\title{
Post-outburst radio observation of the region around McNeil's nebula (V1647 Orionis) (Research Note)
}

\author{
S. Vig ${ }^{1}$, S. K. Ghosh ${ }^{1}$, V. K. Kulkarni ${ }^{2}$, and D. K. Ojha ${ }^{1}$ \\ 1 Tata Institute of Fundamental Research, Mumbai 400 005, India \\ e-mail: sarita@tifr.res.in \\ 2 National Centre for Radio Astrophysics, Pune 411 007, India
}

Received 11 May 2005 / Accepted 10 October 2005

ABSTRACT

We present post-outburst ( $\sim 100$ days after outburst) radio continuum observation of the region $\left(\sim 30^{\prime} \times 30^{\prime}\right)$ around McNeil's nebula (V1647 Orionis). The observations were carried out using the Giant Metrewave Radio Telescope (GMRT), India, at 1272 MHz on 2004 Feb. 14.5 UT. Although 8 sources have been detected within a circular diameter of $25^{\prime}$ centred on V1647 Ori, we did not detect any radio continuum emission from McNeil's nebula. We assign a $5 \sigma$ upper limit of $0.15 \mathrm{mJy} /$ beam for V1647 Ori where the beam size is $5.6^{\prime \prime} \times 2.7^{\prime \prime}$. Even at higher frequencies of 4.9 and $8.5 \mathrm{GHz}$ (VLA archival data), no radio emission has been detected from this region. Three scenarios, namely emission from a homogeneous HII region, ionised stellar wind and shock-ionised gas, are explored in the light of our GMRT upper-limit. For the case of a homogeneous HII region, the radius of the emitting region is constrained to be $\$ 26 \mathrm{AU}$ corresponding to a temperature $\gtrsim 2500 \mathrm{~K}$, which is consistent with the reported radio and $\mathrm{H} \alpha$ emission. In the ionised stellar wind picture, our upper limit of radio emission translates to $\dot{M} / v_{\infty}<1.2-1.8 \times 10^{-10} M_{\odot} \mathrm{yr}^{-1} \mathrm{~km}^{-1} \mathrm{~s}$. On the other hand, if the stellar wind shocks the dense neutral (molecular) cloud, the radio upper limit implies that the fraction of the wind encountering the dense obstacle is $<50 \%$. Based on a recent measurement of X-ray outburst and later monitoring, the expected radio emission has been estimated. Using our radio limit, the radius $(\$ 36 \mathrm{AU})$ and electron density $\left(\gtrsim 7.2 \times 10^{7} \mathrm{~cm}^{-3}\right)$ of the radio emitting plasma have been constrained using a two phase medium in pressure equilibrium for a volume filling factor of 0.9 .

Key words. stars: formation - stars: individual: V1647 Orionis - radio continuum: stars - stars: circumstellar matter

\section{Introduction}

McNeil, in January 2004, reported the presence of a new reflection nebula, now known as McNeil's nebula, in the Lynds dark cloud 1630 in Orion B molecular complex (McNeil 2004). McNeil's nebula (V1647 Orionis) is at a distance of $400 \mathrm{pc}$ (Anthony-Twarog 1982) and is positionally coincident to the infrared source IRAS 05436-0007. Reipurth \& Aspin (2004) presented the optical and near IR photometry of the source V1647 Ori (on 2004 Feb. 14 and Feb. 3 UT, respectively), and found that the star brightened by about 3 mag in the near infrared $(J, H, K)$ with respect to 2MASS measurements (1998). The P-Cygni profile of the $\mathrm{H} \alpha$ line emission from the region around this source implies considerable mass loss in powerful winds with velocities reaching upto $\sim 600 \mathrm{~km} \mathrm{~s}^{-1}$ (Reipurth $\&$ Aspin 2004). Briceño et al. (2004) constrained the epoch of the onset of the outburst to be within 28 Oct. -15 Nov., 2003 by means of $I$ band images of this region. Optical and near infrared photometry of the exciting star, V1647 Ori, show that it is variable atop a general decline in brightness of about 0.3-0.4 mag in 87 days, between 2004, February 10 and May 7 (Walter et al. 2004). Vacca et al. (2004) have presented the near infrared spectroscopy at medium resolution of the McNeil's nebular object after the outburst on March 9, 2004 UT. They suggest it to be a low mass Class I protostar with mass outflow $\left(4 \times 10^{-8} M_{\odot} / \mathrm{yr}\right)$ occuring in a dense ionised wind of velocity $\sim 400 \mathrm{~km} \mathrm{~s}^{-1}$. Andrews et al. (2004) show that the $12 \mu \mathrm{m}$ flux of this source increased by a factor of $\sim 25$ after the outburst whereas the submillimeter continuum is at the preoutburst level.

Kastner et al. (2004) observed a surge in X-ray brightness coincident with the optical and near infrared eruption, and suggest that it could be due to a sudden onset of the rapid accretion phase. More recent X-ray observations by Grosso et al. (2005) have led to detection of $\mathrm{X}$-ray variability within a $\sim 12 \mathrm{~h}$ duration (2004 April 4). They conclude that the soft X-rays originate from accretion shock onto the photosphere of a low mass star and the harder component is due to magnetic reconnection events. Enhanced accretion events from a circumstellar accretion disk are believed to be responsible for such outbursts in young stars. EX Lupi - like objects (EXors) and FU Orionis objects (FUors) are classes of optically variable young stellar objects associated with eruptions. EXor eruptions are generally shorter (few months to a few years) than FUor brightenings (which last many decades). While Walter et al. (2004) 
suggest that the McNeil's nebula is an FUor - like outburst, McGehee et al. (2004) from optical and near infrared measurements infer that the brightening is suggestive of an EXor - like outburst. Ábrahám et al. (2004) compiled the infrared-submmmm spectral energy distribution of the outburst star in its quiescent phase and find that it resembles those of FUor objects indicating the presence of a circumstellar envelope. Ojha et al. (2005) have presented the short term variability as well as the near infrared morphology of McNeil's nebula and showed a significant variation in the source brightness $(>0.15 \mathrm{mag}$ ) within a period of one week. Rettig et al. (2005) have presented high resolution infrared spectra of V1647 Ori. They suggest that the $\mathrm{CO}$ emission lines are likely to originate from $\sim 2500 \mathrm{~K}$ gas in the inner accretion disk region.

Young stellar objects in their pre-main sequence stage generally have very weak radio emission. Although $\operatorname{Br} \gamma$ and $\mathrm{H} \alpha$ lines, which trace the hot ionised gas, have already been detected in McNeil's nebula, no radio study exists in the literature. Hence, we carried out radio observations of McNeil's nebula on 2004 Feb. 14 UT ( 22 days after the first announcement by McNeil on 2004 Jan 23 UT; 91-109 days after the outburst as inferred by Briceño et al. 2004) using the Target of Opportunity (ToO) allocation of the observing time on the Giant Metrewave Radio Telescope (GMRT). In this paper, we present the radio measurement of the region around McNeil's nebula, carried out at $1272 \mathrm{MHz}$. We explore three possible scenarios to understand the radio emission each involving thermal bremstrahlung from the ionised gas. Section 2 describes the observations and data reduction. Sect. 3 gives the results; in Sect. 4, radio emission from three scenarios, viz. a homogeneous HII region, ionised stellar wind and shock ionised region have been considered. Physical constraints on the emitting region based on radio and other available measurements ( $\mathrm{H} \alpha$ and $\mathrm{X}$-ray) have also been discussed in this section. In Sect. 5, we present our conclusions.

\section{Observations and data reduction}

The radio continuum interferometric observations of V1647 Ori were carried out at $1272 \mathrm{MHz}$ on 2004, Feb. 14.5 UT using the GMRT located at Khodad $\left(19^{\circ} 5^{\prime} 30^{\prime \prime} \mathrm{N}, 74^{\circ} 3^{\prime} 0^{\prime \prime} \mathrm{E}\right)$ in India. GMRT has a "Y" shaped hybrid configuration of 30 antennas, each antenna of 45-m diameter. Of the 30 antennas, six each are placed along the east, west and south arms (arm length $\sim 14 \mathrm{~km}$ ). The longest interferometric baseline among the most distant antennas is about $25 \mathrm{~km}$. The remaining twelve are located randomly in a compact $1 \times 1 \mathrm{~km}^{2}$ at the center (Swarup et al. 1991). The phase center of the observations was $(\alpha, \delta)_{2000}=\left(05^{\mathrm{h}} 46^{\mathrm{m}} 14.1^{\mathrm{s}},-00^{\circ} 05^{\prime} 48^{\prime \prime}\right)$, close to the centre of the diffuse optical emission (Reipurth \& Aspin 2004). The total duration of the observations was $3 \mathrm{~h}$. The standard flux calibrator 3C48 was observed at the beginning and end of the observations. The QSO B0500+019 was used for phase calibration. The continuum observations were carried out with a $16 \mathrm{MHz}$ bandwidth.

The NRAO Astronomical Image Processing System (AIPS) was used for the reduction of data. Before analysing the data, it was carefully checked for radio interference and other instrumental problems and the corrupted data were removed. The amplitude and phase calibrated data were Fourier transformed to make images which were then deconvolved using the IMAGR task in AIPS. This task uses the CLEAN algorithm. The sky was approximated by a 2-dimensional plane ( $\sim 8^{\prime}$ from phase center) close to the phase tracking center. However, since a region of $\sim 30^{\prime} \times 30^{\prime}$ has been considered, a number of such 2-dimensional fields have been considered for the Fourier transformation and deconvolution. These fields were projected onto a single tangent plane on the phase center using the AIPS task FLATN. Self calibration was carried out using the task CALIB to fix the final phases and obtain the improved final maps. Considering that a region larger than the primary beam $\left(\sim 21^{\prime}\right)$ has been cleaned, the task PBCOR was used to apply the primary beam correction to the image. For the sources detected in the radio map, the peak position as well as the flux densities (peak and integrated) were obtained using the task JMFITS in AIPS.

We have also analysed the data for V1647 Ori at 4.9 and 8.5 GHz taken from the NRAO Data Archive ${ }^{1}$ obtained using the Very Large Array (VLA) in the B/C configuration. The observation was carried out on March 05, 2005. Since the VLA datasets did not include any of the standard flux calibrators, the VLA phase calibrator, 0541-056, was used for both the phase and flux calibration. A method of analysis, in AIPS, similar to that described above was used for the reduction of this data.

\section{Results}

A map of nearly $30^{\prime} \times 30^{\prime}$ at $1272 \mathrm{MHz}$ has been generated, centred on V1647 Ori. Emission from a part of this region $\left(12^{\prime} \times 12^{\prime}\right)$ around V1647 Ori is shown in Fig. 1. The position of V1647 Ori is marked by a "+" symbol. The synthesized beam size is $5.6^{\prime \prime} \times 2.7^{\prime \prime}$ with position angle $=-48.9^{\circ}(F W H M$ along the major axis corresponds to $2234 \mathrm{AU}$ for $d=400 \mathrm{pc}$ ). The rms noise in our map is $\sim 30 \mu \mathrm{Jy} /$ beam. No radio emission has been detected in our map at the location of V1647 Ori or around $\left(\sim 2^{\prime}\right)$ it. We place a fairly strict $5 \sigma$ upper limit of $0.15 \mathrm{mJy} /$ beam for the radio emission from this source. Away from the McNeil's nebular region, eight radio sources were clearly detected on the map. The coordinates as well as the integrated flux densities of these sources are presented in Table 1. The integrated flux densities of the sources are in the range 0.94-8.42 mJy. At this bright level, there are several sources detected by the NRAO VLA Sky Survey (NVSS) at $1400 \mathrm{MHz}$, which have also been listed in Table 1. Seven of the eight sources detected by us in a circular region of diameter $25^{\prime}$ appear in the NVSS catalog. The faintest one among the sources detected in our image, labelled by identification number 4 in Fig. 1, does not appear in the NVSS catalog. The separations in the positions of these sources between our GMRT map and the NVSS catalog are in the range of $1.8^{\prime \prime}-5.2^{\prime \prime}$ which is reasonable considering the larger synthesized beam for $\operatorname{NVSS}\left(\sim 45^{\prime \prime}\right)$.

1 The National Radio Astronomy Observatory is a facility of the National Science Foundation operated under cooperative agreement by Associated Universities, Inc. 


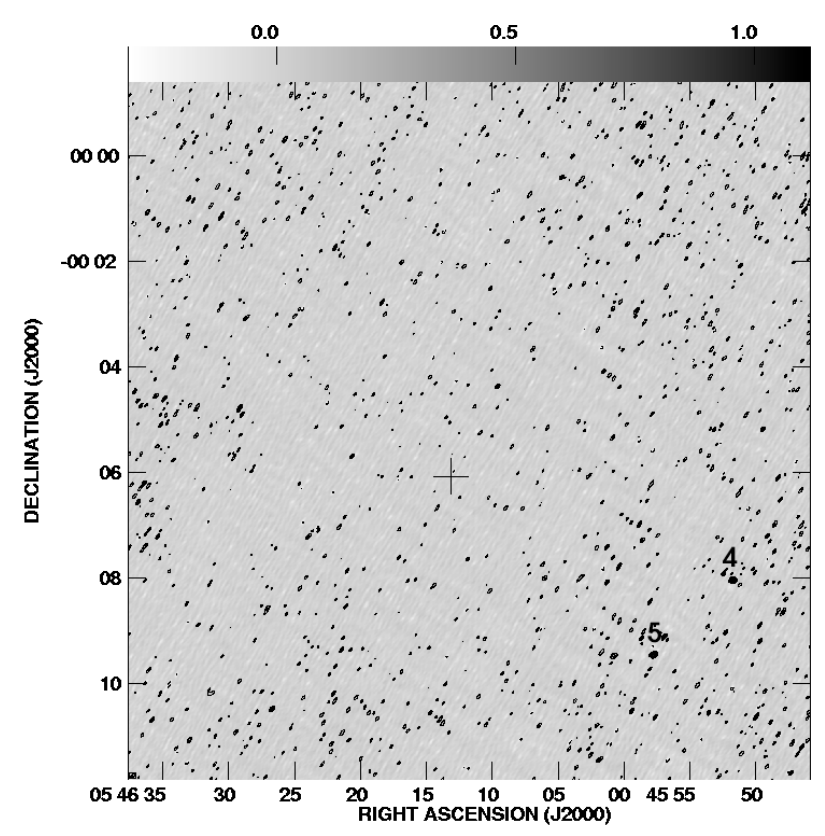

Fig. 1. Radio continuum emission at $1272 \mathrm{MHz}$ from the region around V1647 Ori (McNeil's nebula) and sources with ID 4 and 5. The position marked by a "+" symbol represents the location of V1647 Ori $\left[\alpha_{2000}, \delta_{2000}=\left(05^{\mathrm{h}} 46^{\mathrm{m}} 13.14^{\mathrm{s}},-0^{\circ} 06^{\prime} 04.8^{\prime \prime}\right)\right]$. The synthetic beam size is $5.6^{\prime \prime} \times 2.7^{\prime \prime}$ and the rms noise in the map is $\sim 30 \mu \mathrm{Jy} / \mathrm{beam}$. The contour levels are at $90 \times(-2,1,2,3,4,5,6) \mu \mathrm{Jy} /$ beam.

The identification (ID) numbers of the sources (Table 1) have been used to label them in Fig. 1.

Maps of the region around V1647 Ori at 4.9 and $8.5 \mathrm{GHz}$ were generated using the VLA data. The rms noise in the maps were 25 and $18 \mu \mathrm{Jy} /$ beam and the synthesized beam sizes are $4.8^{\prime \prime} \times 4.0^{\prime \prime}$ and $6.6^{\prime \prime} \times 2.3^{\prime \prime}$ at 4.9 and $8.5 \mathrm{GHz}$, respectively. No radio emission has been detected at the position of V1647 Ori in these maps at either frequency and we place $5 \sigma$ upper limits of 125 and $90 \mu \mathrm{Jy} /$ beam at 4.9 and $8.5 \mathrm{GHz}$, respectively. In what follows, the limit obtained from GMRT measurements has been used to quantitatively constrain the physical parameters of the region around V1647 Ori. The upper limits at 4.9 and $8.5 \mathrm{GHz}$ could be uncertain by a factor of up to $\sim 1.3$ and 2 , respectively, due to variability of the calibrator (Tornikoski et al. 2001). In spite of this, the limits at higher frequencies from VLA would also support similar conclusions based on limits at $1272 \mathrm{MHz}$.

\section{Discussion}

Next, we explore possible scenarios and discuss the implications of our upper limit on radio emission from V1647 Ori. It is believed that the source of outburst, V1647 Ori, is a very young low mass stellar object (Vacca et al. 2004; Ábrahám et al. 2004). In the literature, a number of suggestions have been made about radio emission from embedded low-mass young stellar objects (Gibb 1999).

The radio emission could arise from (a) shocks in HerbigHaro flows (Curiel et al. 1993), (b) an ionised stellar wind/jet (Martin 1996) (c) the ionised region surrounding a protostellar accretion shock (Neufield \& Hollenbach 1996), or (d) magnetic fields close to the star giving rise to a star-disk interaction (André 1996). For V1647 Ori, we explore the possibilities of thermal radio emission from a homogeneous HII region, ionised stellar winds and shocks by outflows.

\subsection{Homogeneous HII region}

Here, we consider radio emission from a homogeneous HII region around the young stellar object V1647 Ori. In this scenario, we assume that the radio emitting region is (i) spherically symmetric and of constant density; (ii) pure hydrogen and dust free; (iii) isothermal; (iv) identical to the $\mathrm{H} \alpha$ emitting region and optically thin in $\mathrm{H} \alpha$ (case A of Osterbrock 1989). The measured $\mathrm{H} \alpha$ emission and the radio upper limit are used to constrain physical parameters like density and size of the emitting region for an assumed temperature. In the case of a low-mass stellar object like V1647 Ori, a possible source of ionising radiation (ultraviolet photons) could be the boundary layer between the stellar surface and the accretion disk (Hartmann 1998).

Using the normalised $\mathrm{H} \alpha$ profile of V1647 Ori obtained by Walters et al. (2004) on Feb. 13, 2004, from a slit whose smaller side corresponds to $600 \mathrm{AU}(d=400 \mathrm{pc})$ and their calibrated photometry in the $B, V, R$ and $I$ bands, we obtain the peak of the $\mathrm{H} \alpha$ emission to be $9.7 \times 10^{-15} \mathrm{erg} \mathrm{cm}^{-2} \mathrm{~s}^{-1} \AA^{-1}$. The total integrated and dereddened $\mathrm{H} \alpha$ emission from the slit using $A_{\mathrm{V}}=11$ (from Vacca et al. 2004) and the extinction law of Rieke \& Lebofsky (1985) is $4.2 \times 10^{-10} \mathrm{erg} \mathrm{cm}^{-2} \mathrm{~s}^{-1}$. According to Retigg et al. (2005), the temperature of the gas in the inner accretion disk of V1647 Ori is $\sim 2500 \mathrm{~K}$ based on the CO emission lines in the infrared. Vacca et al. (2004) interpret the Brackett transitions to be emitted from a gas of temperature $10000 \mathrm{~K}$. We therefore consider temperatures of $2500 \mathrm{~K}, 5000 \mathrm{~K}$ and $10000 \mathrm{~K}$ for our discussion here. For various radii of the emitting region, we determine the electron densities consistent with the measured $\mathrm{H} \alpha$ emission using the recombination coefficients given by Osterbrock (1989). These electron densities have been used to determine the total radio flux at $1272 \mathrm{MHz}$ using the formulation of Spitzer (1978).

Figure 2 shows the relation between electron density and various radii of the emitting region (in AU). These curves, labelled as "H $\alpha$ and Radio" in the figure are presented for three temperatures $2500-10000 \mathrm{~K}$. The solid line intersecting the curves for different temperatures represents the radio flux limit of $150 \mu \mathrm{Jy}$. The region below this line and along the curves represents the allowed parameter space. Here, for a given temperature, the pair of radius and electron density gives rise to consistent values of $\mathrm{H} \alpha$ emission and the radio upper limit. The solid line corresponds to an emitting region (radius) of 13-26 AU for 10000-2500 K. For the same temperature range, the electron densities vary as $3.5 \times 10^{7}-6.5 \times 10^{6} \mathrm{~cm}^{-3}$, respectively.

\subsection{Ionised stellar wind}

We next explore radio emission from an ionised stellar wind. There is an indication of strong stellar winds from the outburst source V1647 Ori (P-Cygni profiles observed in the $\mathrm{H} \alpha$ line; Vacca et al. 2004). The radio emission from ionised stellar 
Table 1. Radio sources detected in a circular region of diameter 25' around McNeil's nebula.

\begin{tabular}{|c|c|c|c|c|c|c|c|c|}
\hline & \multicolumn{3}{|c|}{ 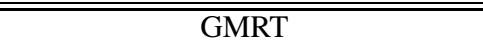 } & \multicolumn{3}{|c|}{ 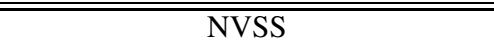 } & \multicolumn{2}{|c|}{ 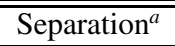 } \\
\hline ID no. & $\begin{array}{c}\text { RA (J2000) } \\
\text { h m s }\end{array}$ & $\begin{array}{c}\operatorname{Dec}(2000) \\
\circ, 1,\end{array}$ & $\begin{array}{c}\text { Int flux } \\
\text { mJy }\end{array}$ & $\begin{array}{c}\text { RA (J2000) } \\
\text { h m s }\end{array}$ & $\begin{array}{c}\text { Dec (J2000) } \\
\circ,{ }^{\prime}\end{array}$ & $\begin{array}{c}\text { Int flux } \\
\text { mJy }\end{array}$ & $\begin{array}{l}\Delta \alpha \\
\prime\end{array}$ & $\begin{array}{l}\Delta \delta \\
\prime \prime\end{array}$ \\
\hline 1 & 054527.26 & -000547.7 & 2.55 & 054527.46 & -000547.6 & 7.5 & -3.0 & -0.1 \\
\hline 2 & 054539.42 & 000500.2 & 8.41 & 054539.64 & +000458.1 & 23.9 & -3.3 & 2.1 \\
\hline 3 & 054543.93 & -001356.5 & 2.24 & 054543.94 & -00 1400.1 & 2.9 & -0.15 & 3.6 \\
\hline 4 & 054551.72 & -000803.0 & 0.94 & - & - & - & - & - \\
\hline 5 & 054557.71 & -000927.8 & 1.24 & 054557.49 & -000924.5 & 2.6 & 3.3 & -3.3 \\
\hline 6 & 054629.53 & -001941.4 & 4.88 & 054629.80 & -00 1944.4 & 15.5 & -4.1 & 3.0 \\
\hline 7 & 054640.76 & -00 1823.8 & 8.42 & 054640.80 & -00 1825.5 & 12.0 & -0.6 & 1.7 \\
\hline 8 & 054643.98 & 000141.7 & 4.59 & 054644.07 & +000144.8 & 5.9 & -1.4 & -3.1 \\
\hline
\end{tabular}

${ }^{a}$ GMRT - NVSS.

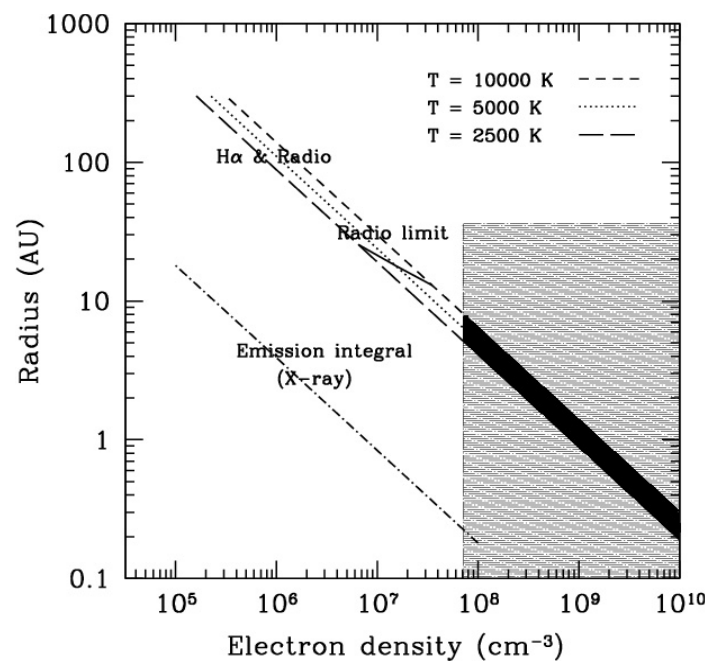

Fig. 2. The radius of the emitting region as a function of electron density are plotted for emission from a homogeneous HII region consistent with radio and $\mathrm{H} \alpha$ measurements (for 2500,5000 and $10000 \mathrm{~K}$ ). The solid line corresponds to the radio upper limit of $0.15 \mathrm{mJy}$. The dash-dotted line represents the constraints from the X-ray emission integral, $n_{\mathrm{e}}^{2} V$, (HIGH state; see Sect. 4.4). The striped region represents the allowed values when the $\mathrm{X}$-ray and radio emitting regions are in a two-phase plasma model in pressure equilibrium for a volume filling factor of 0.1 for the hotter plasma. The dark region includes the $\mathrm{H} \alpha$ constraints also.

winds of young stellar objects has been discussed by Panagia (1991). Under the simplistic assumptions that the stellar wind is (a) fully ionised; (b) spherically symmetric with constant massloss rate and velocity; (c) isothermal with the temperature of stellar wind being the same as that of stellar photosphere; and (d) is in quasi-LTE conditions, the monochromatic radio flux density is given by (Panagia \& Felli 1975)

$$
\begin{aligned}
\frac{S_{v}}{\mathrm{mJy}}= & 5.12\left[\frac{v}{10 \mathrm{GHz}}\right]^{0.6}\left[\frac{T}{10^{4} \mathrm{~K}}\right]^{0.1}\left[\frac{\dot{M} / 10^{-6} \frac{M_{\odot}}{\mathrm{yr}}}{v_{\infty} / 100 \mathrm{~km} \mathrm{~s}^{-1}}\right]^{4 / 3} \\
& \times\left[\frac{d}{\mathrm{kpc}}\right]^{-2}
\end{aligned}
$$

where $S_{v}$ is the expected radio flux density at frequency $v$ from the ionised stellar wind, $T$ is the temperature, $\dot{M}$ is the mass-loss rate, $v_{\infty}$ is the terminal velocity of the stellar wind, and $d$ is the distance to the source. Temperatures of isothermal stellar winds vary between $3 \times 10^{3} \mathrm{~K}$ to $3 \times 10^{5} \mathrm{~K}$ for stars of mass $1 M_{\odot}$ (Lamers and Cassinelli 1999). For this temperature range, our upper limit of radio emission implies the ratio of mass-loss rate to velocity of the stellar wind, $\left(\dot{M} / v_{\infty}\right)$, to be $<1.2-1.8 \times 10^{-10} M_{\odot} \mathrm{yr}^{-1} \mathrm{~km}^{-1} \mathrm{~s}$. Using the inferred massloss rate $\left(\sim 4 \times 10^{-8} M_{\odot} \mathrm{yr}^{-1}\right)$ and wind velocity $\left(\sim 400 \mathrm{~km} \mathrm{~s}^{-1}\right)$ from the P-Cygni profile of the $\mathrm{H} \alpha$ emission by Vacca et al. (2004), we find that $\dot{M} / v_{\infty}=1 \times 10^{-10} M_{\odot} \mathrm{yr}^{-1} \mathrm{~km}^{-1} \mathrm{~s}$, which is consistent with our upper limit.

In order to explain the $\mathrm{H} \alpha$ measurement (Sect. 4.1) for $T=$ $10000 \mathrm{~K}$ and using the density determined from the mass-loss rate of a spherical stellar wind close to the stellar surface for a $1 R_{\odot}$ star, the emission should be in the form of an extremely narrow bipolar flow of solid angle $4 \times 10^{-10} \mathrm{sr}$, which appears unphysical.

\subsection{Shock-ionised gas}

We consider shocks by outflows as a possible source of radio emission from V1647 Ori although the near infrared $\left(\mathrm{H}_{2}\right)$ and optical ([SII]) studies by Reipurth \& Aspin (2004) and Vacca et al. (2004) showed no evidence of shocked gas. In addition, the star associated with McNeil's nebula was proposed as the exciting source of HH23 (Eislöffel \& Mundt 1997; Kastner et al. 2004). Shocks could form due to a supersonic stellar wind impinging upon a dense clump of gas in the flow close to the exciting source (Hartigan et al. 1987). Fast shocks (shock velocity $>60 \mathrm{~km} \mathrm{~s}^{-1}$ ) due to winds from young stellar objects into molecular clouds are a potential source of free-free radio emission since the shock energy ionises the compressed gas. If a stellar wind with a mass-loss rate of $\dot{M}$ and terminal velocity $v_{\infty}$ is shocked due to the presence of an obstacle such as a molecular cloud, then the radio continuum flux density, $S_{v}$, in the optically thin limit is given by (Curiel et al. 1989)

$$
\begin{aligned}
\frac{S_{v}}{\mathrm{mJy}}= & 3.98 \times 10^{-2} \eta\left[\frac{\dot{M}}{10^{-7} M_{\odot} \mathrm{yr}^{-1}}\right]\left[\frac{v_{\infty}}{100 \mathrm{~km} \mathrm{~s}^{-1}}\right]^{0.68} \\
& \times\left[\frac{d}{\mathrm{kpc}}\right]^{-2}\left[\frac{T}{10^{4} \mathrm{~K}}\right]^{0.45}\left[\frac{v}{5 \mathrm{GHz}}\right]^{-0.1}
\end{aligned}
$$

where $\eta$ represents the geometrical fraction of the stellar wind that is shocked, $d$ is the distance to the source, $T$ is the 
temperature and $v$ is the frequency. For a temperature of $10000 \mathrm{~K}$, and using the values of distance, mass-loss rate and velocity of stellar wind of V1647 Ori given in Sect. 4.2, we find that the expected radio flux is $300 \eta \mu \mathrm{Jy}$. Hence, our limit implies that if the shocked ionised gas is responsible for the radio emission, the fraction of the wind encountering the dense molecular obstacle is $\eta<50 \%$.

\subsection{X-ray measurements and its implications}

Recently, an X-ray outburst has been detected from V1647 Ori, which illuminates the McNeil's nebula, by Kastner et al. (2004) based on their observations using the Chandra satellite. Their reported observations correspond to three epochs (14 Nov. 2002, 7 March 2004 and 22 March 2004) showing quiescent (LOW), outburst (HIGH) and post-outburst emission. Thereafter Grosso et al. (2005) have detected enhanced X-ray variability from a long integration using the XMM-Newton satellite on 4 April 2004. Kastner et al. (2004) have modelled their observations and concluded that the plasma responsible for the X-ray emission during the HIGH state is characterised by a temperature $T_{\mathrm{X}} \sim 5.6 \times 10^{7} \mathrm{~K}$ and $\mathrm{X}$-ray luminosity $L_{\mathrm{X}} \sim 10^{31} \mathrm{erg} \mathrm{s}^{-1}$. The corresponding values for $T_{\mathrm{X}}$ and $L_{\mathrm{X}}$ during the LOW state are $\sim 10^{7} \mathrm{~K}$ and $\sim 3.0 \times 10^{29} \mathrm{erg} \mathrm{s}^{-1}$ respectively. The XMM-Newton data imply slightly different values $\left(T_{\text {hard }} \sim 4.94 \times 10^{7} \mathrm{~K}, T_{\text {soft }} \sim 1.06 \times 10^{7} \mathrm{~K}, L_{\mathrm{X}} \sim\right.$ $1-3 \times 10^{31} \mathrm{erg} \mathrm{s}^{-1}$; Grosso et al.). They propose that while the soft X-rays could originate from accretion shock onto the photosphere of a low mass star, the harder component is likely to be due to magnetic reconnection events. Here, we consider the implications on expected radio emission at $1272 \mathrm{MHz}$ based on the Chandra measurements.

The X-ray emission from hot plasmas is well quantified in terms of "band cooling coefficients" in different energy ranges as a function of temperature. We have used the band cooling coefficients calculated by Raymond et al. (1976). Considering the Chandra X-ray band to be $0.5-8 \mathrm{keV}$, we obtain the emission integral, $n_{\mathrm{e}}^{2} V$ (where $n_{\mathrm{e}}$ is the electron density and $V$ is the volume occupied by the plasma), to be $8.6 \times 10^{52} \mathrm{~cm}^{-3}$ during the LOW state and $8.3 \times 10^{53} \mathrm{~cm}^{-3}$ during the HIGH state. Assuming a spherical emitting region of constant density, the above values constrain the radius, $R$ and $n_{\mathrm{e}}$ for the HIGH state as shown by a dash-dotted line in Fig. 2 .

We estimate the expected radio emission from the X-ray emitting plasma, assuming it to be optically thin, following Spitzer (1978). They are 0.01 and $0.05 \mu \mathrm{Jy}$ corresponding to the LOW and HIGH states respectively (for $d=400 \mathrm{pc}$ ). These values are much smaller than (and hence consistent with) our upper limit at $1272 \mathrm{MHz}$. If the assumption of optical thinness does not hold, then the emission would be even lower.

Next, we consider the hot plasma to be clumpy with two components in pressure equilibrium, the hotter component (electron density $n_{1}$, temperature $T_{1}$ ) responsible for the X-ray emission and the other $\left(n_{2}, T_{2}\right)$ component for the radio. The pressure equilibrium implies $n_{1} T_{1}=n_{2} T_{2}$. Let the volume filling factor for the hotter component be $f=\left(V_{\mathrm{X} \text {-ray }} /\left(V_{\mathrm{X} \text {-ray }}+\right.\right.$ $\left.V_{\text {radio }}\right)$ ). If $f \ll 1$, then $f \sim\left(V_{\mathrm{X}-\text { ray }} / V_{\text {radio }}\right)$. Assuming

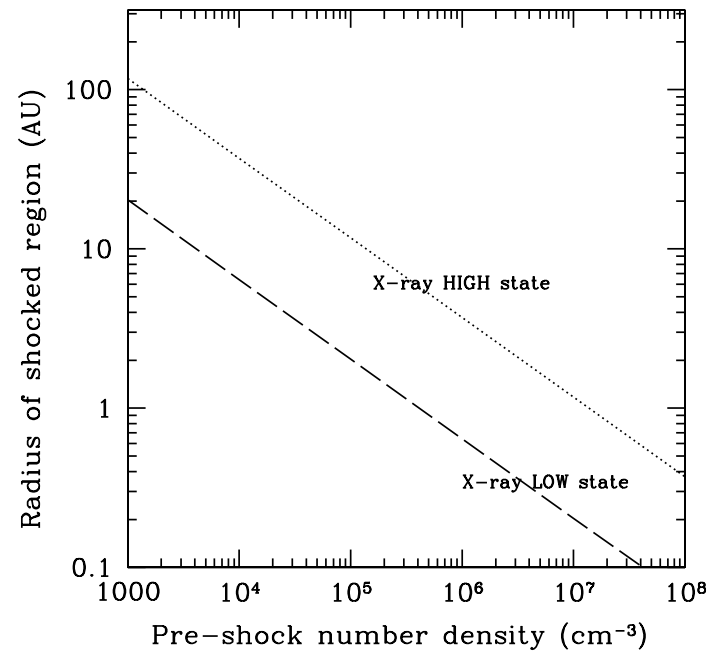

Fig. 3. Constraints on the size of X-ray emitting shocked region and pre-shock number density for V1647 Ori from measured X-ray luminosities. The dotted and dashed line refer to the HIGH and LOW state of X-ray emission, respectively.

$T_{2}=5000 \mathrm{~K}$, we estimate the $n_{\mathrm{e}}^{2} V$ for the radio emitting gas corresponding to both the states, which we translate to radio flux density for the optically thin case. The values turn out to be rather large, viz., $(2.0 / f)$ and $(607 / f)$ Jy corresponding to LOW and HIGH states respectively. Both are much larger than our limit of $150 \mu \mathrm{Jy}$. If the non-detection of V1647 Ori at $1272 \mathrm{MHz}$ is purely due to optical depth effects, we can constrain the size, $R$, and density, $n_{\mathrm{e}}$, of the radio emitting phase using the upper limit in this two phase plasma scenario. We find $R \lesssim 36$ AU and $n_{\mathrm{e}} \gtrsim 7.2 \times 10^{7} \mathrm{~cm}^{-3}\left(1.3 \times 10^{9} \mathrm{~cm}^{-3}\right)$ for the LOW (HIGH) state, corresponding to a volume filling factor, $f=0.1$, which is depicted in Fig. 2 by the striped region.

In ionised stellar wind picture, the X-ray emission considering that (i) the $\mathrm{X}$-ray luminosity is dominated by the free-free emission of hydrogen (ii) $T=10000 \mathrm{~K}$, and (iii) density determined from mass-loss rate of a spherical stellar wind close to the stellar surface for a $1 R_{\odot}$ star, is $<10^{24} \mathrm{erg} \mathrm{s}^{-1}$, much lower than that measured by Chandra.

In Sect. 4.3, we have considered shocked gas as a source of radio emission. Here, we extract physical parameters of this scenario from the X-ray measurements using the prescription of Raga et al. (2002). In this scheme, a bow shock is created at a thin surface near the apex of a dense, approximately spherical obstacle of radius $r_{\mathrm{b}}$ which emits X-ray of luminosity, $L_{\mathrm{X}}$, given by

$\frac{L_{\mathrm{X}}}{L_{\odot}}=4.1 \times 10^{-6}\left(\frac{n_{0}}{100 \mathrm{~cm}^{-3}}\right)\left(\frac{r_{\mathrm{b}}}{10^{16} \mathrm{~cm}}\right)^{2}\left(\frac{v_{\mathrm{s}}}{100 \mathrm{~km} \mathrm{~s}^{-1}}\right)^{5.5}$.

Here, $n_{0}$ is the pre-shock number density and $v_{\mathrm{s}}$ is the shock velocity. Taking the X-ray luminosity of V1647 Ori from Chandra measurements (Sect. 4.4) and $v_{\mathrm{s}}=400 \mathrm{~km} \mathrm{~s}^{-1}$, we obtain the size of the X-ray emitting shocked region as a function of $n_{0}$. This variation of density with $r_{\mathrm{b}}$ for the LOW and HIGH states is shown in Fig. 3. 


\section{Conclusions}

In the present study, three scenarios have been explored to explain the radio, $\mathrm{H} \alpha$ and X-ray measurements of McNeil's nebula. For the case of homogeneous HII region, the size of the emitting region and the electron density must lie in the darkshaded region of Fig. 2 (2500-10000 K) in order to be consistent with all the three measurements. A typical solution would be for $T=5000 \mathrm{~K}$ and $n_{\mathrm{e}}=7.2 \times 10^{7} \mathrm{~cm}^{-3}$; the size and radio flux are then given by $6.3 \mathrm{AU}$ and $19 \mu \mathrm{Jy}$, respectively. In the ionised stellar wind picture, the expected X-ray emission is very small. The $\mathrm{H} \alpha$ measurement also cannot be explained in this scenario. The shocked gas picture can be consistent with X-ray measurements provided the pre-shock number density and the radius of the dense spherical obstacle fall near the curves shown in Fig. 3. However, for these parameters, the $\mathrm{H} \alpha$ measurement cannot be explained.

Although we have considered extremely simple pictures under idealized assumptions, we conclude that the homogeneous HII region scenario explains the radio, $\mathrm{H} \alpha$ as well as $\mathrm{X}$-ray measurements consistently while the ionised stellar wind and shocked gas pictures do not. For the latter two scenarios, we cannot rule out the possibility that these emissions (radio, $\mathrm{X}$-ray and $\mathrm{H} \alpha$ ) are at different length scales. However, in that case, the physical connection implied by the outburst and time variability in all three wavebands will remain unexplained.

Acknowledgements. We thank the anonymous referee for many useful suggestions which have improved the paper. We thank the Centre Director, NCRA-TIFR and members of the Target of Opportunity (ToO) time allotment committee for making time available for our observations. We thank the staff of the GMRT who have made the radio observations possible. GMRT is run by the National Centre for Radio Astrophysics of the Tata Institute of Fundamental Research.

\section{References}

Ábrahám, P., Kóspál, Á., Csizmadia, Sz., et al. 2004, A\&A, 419, L39 André, P. 1996, ASP Conf. Ser., 93, ed. A. R. Taylor, \& J. M. Paredes, 273

Andrews, S. M., Rothberg, B., \& Simon, T. 2004, ApJ, 610, L45
Anthony-Twarog, B. J. 1982, AJ, 87, 1213

Briceño, C., Vivas, A. K., Hernández, J., et al. 2004, ApJ, 606, L123

Curiel, S., Rodriguez, L. F., Canto, J., et al. 1989, Astro. Lett. and Comm., 27, 299

Curiel, S., Rodriguez, L. F., Moran, J. M., \& Canto, J. 1993, ApJ, 415, 191

Eislöffel, J., \& Mundt, R. 1997, AJ, 114, 280

Gibb, A. G. 1999, MNRAS, 304, 1

Grosso, N., Kastner, J. H., Ozawa, H., et al. 2005, A\&A, 438, 159

Hartigan, P., Raymond, J., \& Hartmann, L. 1987, ApJ, 316, 323

Hartmann, L. 1998, Accretion processes in star formation (Cambridge University Press)

Kastner, J. H., Richmond, M., Grosso, N., et al. 2004, Nature, 430, 429

Lamers, H. J. G. L. M., \& Cassinelli, J. P. 1999, Introduction to Stellar Winds (Cambridge University Press)

Martin, S. C. 1996, ApJ, 473, 1051

McGehee, P. M., Smith, J. A., Henden, A. A., et al. 2004, ApJ, 616, 1058

McNeil, J. W. 2004, IAU Circ., 8284

Neufield, D. A., \& Hollenbach, D. J. 1996, ApJ, 471, L45

Ojha, D. K., Kusakabe, N., Tamura, M., et al. 2005, PASJ, 57, 203

Osterbrock, D. E. 1989, Astrophysics of gaseous nebulae and active galactic nuclei (University Science Books)

Panagia, N., \& Felli, M. 1975, A\&A, 39, 1

Panagia, N. 1991, The Physics of Star Formation and Early Stellar Evolution, ed. C. J. Lada, \& N. D. Kylafis (Kluwer Academic Publishers), 565

Raga, A. C., Noriega-Crespo, A., \& Velázquez, P. F. 2002, ApJ, 576, L149

Raymond, J. C., Cox, D. P., \& Smith, B. W. 1976, ApJ, 204, 290

Reipurth, B., \& Aspin, C. 2004, ApJ, 606, L119

Rettig, T. W., Brittain, S. D., Gibb, E. L., Simon, T., \& Kulesa, C. 2005, ApJ, 626, 245

Rieke, G. H., \& Lebofsky, M. J. 1985, ApJ, 288, 618

Spitzer, L. J. 1978, Physical processes in the interstellar medium (John Wiley Publications)

Swarup, G., Ananthakrishnan, S., Kapahi, V. K., et al. 1991, Current Science, 60, 95

Tornikoski, M., Jussila, I., Johansson, P., Leinela, M., \& Valtaoja, E. 2001, AJ, 121, 1306

Vacca, W. D., Cushing, M. C., \& Simon, T. 2004, ApJ, 609, L29

Walter, F. M., Stringfellow, G. Y., Sherry, W. H., \& Field-Pollatou, A. 2004, AJ, 128, 1872 